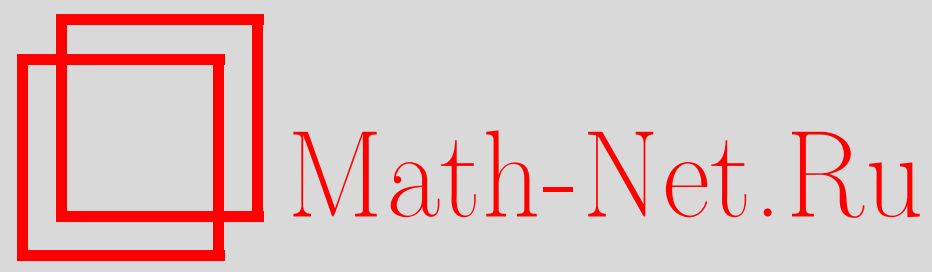

К.-Х. Фихтнер, В. Фройденберг, Ф. Либшер, Расщепления гиббсовских состояний, Матем. заметки, 1998, том 64, выпуск 4, 598-605

DOI: https://doi.org/10.4213/mzm1435

Использование Общероссийского математического портала Math-Net.Ru подразумевает, что вы прочитали и согласны с пользовательским соглашением http://www.mathnet.ru/rus/agreement

Параметры загрузки:

IP : 44.207 .124 .84

26 апреля 2023 г., 15:51:45

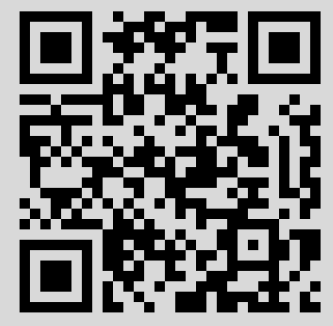




\title{
РАСЩЕПЛЕНИЯ ГИББСОВСКИХ СОСТОЯНИЙ
}

\author{
К.-Х. Фихтнер, В. Фройденберг, Ф. Либшер
}

\begin{abstract}
Обсуждается независимое расщепление состояний, при котором оператор, связанный с расщеплением, оставляет инвариантньм класс равновесных состояний для одномодового электромагнитного поля. Расщепление влияет только на параметры состояния и вызывает диссипацию энергии. После расщепления значения энергии обоих пучков оказываются независимыми. Эта независимость является характеристическим свойством геометрического распределения (распределения значений энергий в состоянии равновесия). Обнаружено, что класс состояний, для которых полные состояния расщепленных частей независимы, образован так называемыми фазовыми состояниями.

Библиография: 17 названий.
\end{abstract}

1. Введение. В статистической механике [1], [2] свойство состояния системы быть равновесным обычно определяется путем изучения распределений в ееподсистемах. Как правило, система считается находящейся в равновесии, если подсистемы имеют тот же тип состояния, но с другим набором термодинамических параметров. Более того, в случае классических систем предполагается, что различные части системы имеют независимые распределения. Если система занимает некоторую область пространства, то ее подсистема обычно задается как часть этой системы, расположенная в подобласти. Однако, для систем частищ возможно также задать подсистему при помощи стохастической процедуры. Например, мы можем случайным образом расщепить конфигурацию системы на две подконфигурации. Для этого каждую частицу независимо от других надо отнести к той или иной подконфигурации (т.е. произвести независимое расщепление). Равновесные состояния систем классических невзаимодействующих частиц и процессы Пуассона [3] характеризуются независимостью двух подконфигураций. При рассмотрении только одной подконфигурации явление расщепления позволяет ввести понятие разрежения.

Наша цель - изучение аналогичной ситуации для случая квантовых бозонных систем. Рассмотрим одну моду квантованного электромагнитного поля. В качестве модельного оператора расщепления пучка в физической литературе [4], [5] обычно используется унитарньй оператор $U_{\tau, \eta}$ такой, что

$$
U_{\tau, \eta} \psi_{z_{1}} \otimes \psi_{z_{2}}=\psi_{\tau z_{1}-\eta z_{2}} \otimes \psi_{\eta z_{1}+\tau z_{2}}
$$

Работа выполнена при частичной поддержке фонда INTAS, грант № 96-0698. 
где $\psi_{z}-$ экспоненциальный вектор, ассоциированньй с $z \in \mathbb{C}$. С этим оператором связан изометрический оператор расщепления

$$
V_{\tau, \eta} \psi_{z}=\psi_{\tau z} \otimes \psi_{\eta z}
$$

Мы можем интерпретировать операцию $\omega \mapsto \omega\left(V_{\tau, \eta}^{*}(\cdot) V_{\tau, \eta}\right)$ на уровне состояний квантовых систем как независимое квантовое расщепление [6], [7]. Это проще всего заметить, рассматривая распределение чисел заполнения [8] (т.е. распределение числа квантов), когда имеет место независимое расщепление.

Процедура разрежения $\omega \mapsto \omega\left(V_{\tau, \eta}^{*}(\cdot \otimes \mathbf{1}) V_{\tau, \eta}\right)$, отображающая любое нормальное состояние в фоковском пространстве в нормальное в том же фоковском пространстве, оказьвается связанной с расщеплением. Если мы начнем с гиббсовского состояния осциллятора (рассматриваемого как состояние теплового равновесия)

$$
\omega^{\beta}(\cdot)=\left(1-e^{-\beta}\right) \operatorname{tr}\left(e^{-\beta N}(\cdot)\right),
$$

где $\beta$ - обратная температура, а $N$ - оператор числа частиц, то получим, что $\omega^{\beta}$ отображается в $\omega^{|\tau|^{-2} \beta}$, т.е. наблюдается уменьшение средней энергии в $|\tau|^{2} \leqslant 1$ раз.

В настоящей работе мы отказываемся от требования независимости и строим пример расщепления, заданного изометрией $\mathscr{V}_{q}$, не являющегося независимым. Соответствующее разрежение также оставляет инвариантньм класс гиббсовских состояний. Точнее, получена формула

$$
\omega^{\beta}\left(\mathscr{V}_{q}^{*}(\cdot \otimes \mathbf{1}) \mathscr{V}_{q}\right)=\omega^{\beta-\ln q}(\cdot) .
$$

Очевидно, что средняя энергия пропорциональна $\beta^{-1}$ и преобразуется нелинейным образом.

Если рассмотреть распределение соответствующих случайных величин (в данной модели это просто распределение уровней энергии), то легко получить следующую характеристику геометрических распределений.

ТЕОРемА. Пусть $\xi, \eta$ - независимые $\mathbb{N}$-значные случайные величины, причем $\eta$ имеет геометрическое распределение. Положим $\xi_{0}=\min (\xi, \eta)$. Тогда $\xi$ имеет геометрическое распределение, если и только если $\xi_{0}$ и $\xi-\xi_{0}$ независимы.

В рамках исходной квантовой модели достаточное условие теоремы может быть интерпретировано как независимость энергий после расщепления.

Мы будем назьвать состояния, возникающие после процедуры расщепления, полностью независимыми. Они, очевидно, аналогичны известным в физической литературе фазовым состояниям [9]. Эти состояния образуют когерентные состояния, обобщенныев смысле Переломова [10], которые связаны с представлением $\mathrm{SU}(1,1)$ при $k=1 / 2$ (представление Хольштейна-Примакова).

2. Основные понятия и определения. Обозначим натуральные, целые, вещественные, положительные вешественные и комплексные числа через $\mathbb{N}=\{0,1,2, \ldots\}, \mathbb{Z}$, $\mathbb{R}, \mathbb{R}_{+}=[0, \infty)$ и $\mathbb{C}$ соответственно, а действительную и мнимую части комплексного числа $z$ через $\operatorname{Re} z$ и $\operatorname{Im} z$.

Рассмотрим пространство $\ell^{2}$ над множеством натуральных чисел:

$$
\Gamma=\left\{\left(c_{n}\right)_{n \in \mathbb{N}}: c_{n} \in \mathbb{C}, \sum_{n \in \mathbb{N}}\left|c_{n}\right|^{2}<\infty\right\}
$$


являющееся канонически изоморфным симметричному фоковскому пространству над $\mathbb{C}$. В качестве ортонормированного базиса используются символы Кронекера $\delta_{n}, n \in \mathbb{N}$. Единичный оператор в пространстве Г обозначим через 1.

В данной работе используется понятие нормального состояния на множестве $\mathfrak{L}(\Gamma)$ всех ограниченных линейных операторов над $\Gamma$. Такие нормальные состояния задаются при помощи отображения $A \mapsto \omega(A)=\operatorname{tr}(\varrho A), A \in \mathfrak{L}(\Gamma)$, где $\varrho$ представляет собой оператор класса $\mathscr{T}_{1}$ над $\Gamma$ со следом $\operatorname{tr} \varrho=1$ и нормой $\|\varrho\|=\operatorname{tr}\left(\varrho^{*} \varrho\right)^{1 / 2}<\infty$, определяющий данное состояние. Он называется матрицей плотности этого состояния.

3. Расщепление, не являющееся независимым. Определим функцию $\alpha_{q}$ : $\mathbb{N}^{2} \rightarrow \mathbb{C}$ по правилу

$$
\alpha_{q}(k, l)= \begin{cases}\sqrt{(1-q) q^{k}}, & \text { если } l \neq 0, \\ \sqrt{q^{k}}, & \text { если } l=0,\end{cases}
$$

для некоторого $0 \leqslant q \leqslant 1$ и построим оператор $\mathscr{V}_{q}: \Gamma \rightarrow \Gamma \otimes \Gamma$ такой, что

$$
\mathscr{V}_{q} \psi(n, m)=\alpha_{q}(n, m) \psi(n+m), \quad \psi \in \Gamma .
$$

Лемма 1. Oператор $\mathscr{V}_{q}$ является изометрическим, а сопряэсенный оператор действует по правилу

$$
\mathscr{V}_{q}^{*} \psi(n)=\sum_{k=0}^{n} \alpha_{q}(k, n-k) \psi(k), \quad \psi \in \Gamma .
$$

ДокАЗАТЕЛьство. Элементарные выкладки дают

$$
\left\|\mathscr{V}_{q} \psi\right\|^{2}=\sum_{n, m \in \mathbb{N}}\left|\alpha_{q}(n, m)\right|^{2}|\psi(n+m)|^{2}=\sum_{k \in \mathbb{N}} \sum_{n=0}^{k}\left|\alpha_{q}(n, k-n)\right|^{2}|\psi(k)|^{2} .
$$

Легко заметить, что $\sum_{n=0}^{k}\left|\alpha_{q}(n, k-n)\right|^{2}=1$ при любых $k \in \mathbb{N}$, так что $\mathcal{V}_{q}$ имеет полную область определения и является изометрическим. Формула для сопряженного оператора получается аналогично.

СлЕДСТВИЕ. Отображения $\omega \mapsto \omega\left(\mathscr{V}_{q}^{*}(\cdot) \mathscr{V}_{q}\right) u \omega \mapsto \omega\left(\mathscr{V}_{q}^{*}(\cdot \otimes \mathbf{1}) \mathscr{V}_{q}\right)$ переводят нормальные состояния на $\mathfrak{L}(\Gamma)$ в нормальные на $\mathfrak{L}(\Gamma) \otimes \mathfrak{L}(\Gamma)$ и $\mathfrak{L}(\Gamma)$ соответственHo.

ЗАмЕчаниЕ 1. Для этих двух отображений мы использовали названия "расщепление" и "разрежение" по аналогии с соответствующими понятиями для точечных случайных распределений [6].

ЗАмЕЧАниЕ 2. Лемма 1 и следствие из нее остаются справедливьги после замены $\alpha_{q}$ на произвольную функцию $\alpha: \mathbb{N}^{2} \rightarrow \mathbb{C}$, удовлетворяющую условию

$$
\sum_{n=0}^{k}|\alpha(n, k-n)|^{2}=1
$$

при любых $k \in \mathbb{N}$. Более того, введенные отображения можно обобщить на случай фоковского пространства над произвольньм пространством квадратично-интегрируемых функций (см. [6] для случая независимых расщеплений). 
4. Связь с гиббсовскими состояниями. Рассмотрим семейство нормальных состояний $\left(\omega^{\beta}\right)_{0<\beta \leqslant \infty}$ на LG $(\Gamma)$, заданных формулой

$$
\omega^{\beta}(A)=\left(1-e^{-\beta}\right) \operatorname{tr}\left(e^{-\beta N} A\right), \quad A \in \mathfrak{L}(\Gamma) .
$$

Таким образом, $N$ есть оператор числа частиц, т.е. $N \delta_{l}=l \delta_{l}$. Вакуумным состоянием является $\omega^{\infty}=\left\langle\delta_{0}, \cdot \delta_{0}\right\rangle$. Параметр $\beta$ интерпретируется как обратная температуpa [11]. Докажем следующее предложение.

ПРЕДЛОЖЕНИЕ 1. При любъ $\beta \geqslant 0, A \in \mathfrak{L}(\Gamma) u 0 \leqslant q \leqslant 1$ uмеeт место тождество

$$
\omega^{\beta}\left(\mathscr{V}_{q}^{*}(A \otimes \mathbf{1}) \mathscr{V}_{q}\right)=\omega^{\beta-\ln q}(A)
$$

ЗАмЕчАниЕ 3. Ввиду того, что величина обратная $\beta$ пропорциональна средней кинетической энергии системы, наблюдается нелинейная диссипация энергии.

ДокАЗАТЕЛЬСТво. Так как $\left(\delta_{n}\right)_{n \in \mathbb{N}}$ является полной ортонормированной системой собственных функций оператора $N$, имеет место

$$
\omega^{\beta}(\cdot)=\left(1-e^{-\beta}\right) \sum_{l=0}^{\infty} e^{-l \beta}\left\langle\delta_{l}, \cdot \delta_{l}\right\rangle .
$$

Положим $\mathscr{V}_{q} \delta_{l}(n, m)=\sqrt{q^{n} b_{q}(m)} \delta_{l}(n+m)$ с коэффициентами

$$
b_{q}(k)= \begin{cases}1-q, & \text { если } k>0, \\ 1, & \text { если } k=0 .\end{cases}
$$

Тогда

$$
\left\langle\mathscr{V}_{q} \delta_{l},(A \otimes \mathbf{1}) \mathscr{V}_{q} \delta_{l}\right\rangle=\sum_{m=0}^{l} q^{l-m} b_{q}(m)\left\langle\delta_{l-m}, A \delta_{l-m}\right\rangle=\sum_{n=0}^{l} q^{n} b_{q}(l-n)\left\langle\delta_{n}, A \delta_{n}\right\rangle .
$$

Суммируя, получаем

$$
\begin{aligned}
\omega^{\beta}\left(\mathscr{V}_{q}^{*}(A \otimes \mathbf{1}) \mathscr{V}_{q}\right) & =\left(1-e^{-\beta}\right) \sum_{l=0}^{\infty} e^{-l \beta}\left\langle\mathscr{V}_{q} \delta_{l},(A \otimes \mathbf{1}) \mathscr{V}_{q} \delta_{l}\right\rangle \\
& =\left(1-e^{-\beta}\right) \sum_{l=0}^{\infty} e^{-l \beta} \sum_{n=0}^{l} q^{n} b_{q}(l-n)\left\langle\delta_{n}, A \delta_{n}\right\rangle \\
& =\left(1-e^{-\beta}\right) \sum_{n=0}^{\infty} \sum_{m=0}^{\infty} e^{-(n+m) \beta} q^{n} b_{q}(m)\left\langle\delta_{n}, A \delta_{n}\right\rangle \\
& =\left(1-e^{-\beta}\right) \sum_{n=0}^{\infty}\left(\sum_{m=0}^{\infty} e^{-m \beta} b_{q}(m)\right) e^{-n \beta} q^{n}\left\langle\delta_{n}, A \delta_{n}\right\rangle
\end{aligned}
$$

С другой стороны,

$$
\sum_{m=0}^{\infty} e^{-m \beta} b_{q}(m)=(1-q) \sum_{m=1}^{\infty} e^{-m \beta}+1=\frac{(1-q) e^{-\beta}+1-e^{-\beta}}{1-e^{-\beta}}=\frac{1-q e^{-\beta}}{1-e^{-\beta}} .
$$

Таким образом,

$$
\omega^{\beta}\left(\mathscr{V}_{q}^{*}(A \otimes \mathbf{1}) \mathscr{V}_{q}\right)=\left(1-q e^{-\beta}\right) \sum_{n=0}^{\infty}\left(q e^{-\beta}\right)^{n}\left\langle\delta_{n}, A \delta_{n}\right\rangle=\omega^{\beta-\ln q}(A) .
$$

Аналогично можно вычислить другое разрежение, заменив $A \otimes \mathbf{1}$ на $\mathbf{1} \otimes A$. 
ПРЕДЛОЖЕНИЕ 2. При любы $\beta \geqslant 0, A \in \mathfrak{L}(\Gamma)$ u $0 \leqslant q \leqslant 1$ имеет место разложсение

$$
\omega^{\beta}\left(\mathscr{V}_{q}^{*}(\mathbf{1} \otimes A) \mathscr{V}_{q}\right)=\frac{1-q}{1-q e^{-\beta}} \omega^{\beta}(A)+\frac{q\left(1-e^{-\beta}\right)}{1-q e^{-\beta}} \omega^{\infty}(A)
$$

ЗАмЕчАниЕ 4. Это расшепление демонстрирует еше один эффект: если одну часть равновесной системы привести в вакуумное состояние, то другая останется без изменений. Изменение средней энергии должно быть линейным несмотря на то, что конечное состояние не является равновесньп.

Используя структуру тензорного произведения произвольного фоковского пространства [12], можно обобшить приведенные выше результаты на случай произвольных симметричных фоковских пространств.

5. Характеризация геометрических распределений. Рассмотрим распределение энергетических уровней в расщепленной системе, если первоначально она находилась в осцилляторном гиббсовском состоянии. Следуя [8], [13], мы используем следующую терминологию. Пусть $\operatorname{Pr}_{n}$ есть проектирование на $\delta_{n}$.

ЛЕмма 2. При любых $k, l \in \mathbb{N}$ справедливо

$$
\mathscr{V}_{q}^{*}\left(\operatorname{Pr}_{k} \otimes \operatorname{Pr}_{l}\right) \mathscr{V}_{q}=q^{k} b_{q}(l) \operatorname{Pr}_{k+l}
$$

ДокАЗАТЕЛьСтво. Возьмем произвольньй вектор $\psi \in \Gamma$. Тогда для $n, k, l \in \mathbb{N}$ имеем

$$
\begin{aligned}
\mathscr{V}_{q}^{*}\left(\operatorname{Pr}_{k} \otimes \operatorname{Pr}_{l}\right) \mathscr{V}_{q} \psi(n) & =\left(\mathscr{V}_{q}^{*} \alpha_{q}(k, l) \psi(k+l) \delta_{k} \otimes \delta_{l}\right)(n) \\
& =\sum_{s=0}^{n} \alpha_{q}(s, n-s) \alpha_{q}(k, l) \psi(k+l) \delta_{k} \otimes \delta_{l}(s, n-s) \\
& =\left|\alpha_{q}(k, l)\right|^{2} \psi(k+l) \delta_{k+l}(n),
\end{aligned}
$$

что завершает доказательство.

Вычисляя математические ожидания, можно получить наглядную интерпретацию этой формулы в терминах случайных натуральных чисел.

ЛЕмма 3. Пусть $\xi, \eta$-независимые случайные челочисленные величины такие, что $\eta$ имеет геометрическое распределение с параметром q. Тогда для любых $k, l \in \mathbb{N}$ выполнено

$$
\mathrm{P}\{\min (\xi, \eta)=k, \xi-\min (\xi, \eta)=l\}=q^{k} b_{q}(l) \mathrm{P}\{\xi=k+l\}
$$

Опишем характеризацию геометрических распределений (см. также [14]).

ТЕОРема 1. Пусть $\xi, \eta$-независимые случайные челочисленные величины, причем $\eta$ имеет геометрическое распределение. Тогда $\xi$ имеет геометрическое распределение тогда и только тогда, когда $\min (\xi, \eta)$ и $\xi-\min (\xi, \eta)$ независимы. 
ЗАмечАниЕ 5. Так как $N$ является осцилляторным гамильтонианом, $\operatorname{Pr}_{k}-$ проекторами на его собственные векторы, то $\widetilde{\omega}\left(\operatorname{Pr}_{k} \otimes \operatorname{Pr}_{l}\right)$ задает распределение энергетических уровней расщепленного состояния $\widetilde{\omega}$. Вместе с леммой 2 данная теорема утверждает, что эти энергетические уровни в разных частях расшепленной системы независимы, если первоначальное состояние представляло собой гиббсовское состояние осциллятоpa.

ДокАЗАТЕЛЬСтво. Необходимость следует из леммы 3 , так как $\mathrm{P}\{\xi=k+l\}$ факторизуется по отношению к $k$ и $l$.

Докажем достаточность. Пусть указанные состояния независимы. Тогда существуют функции $f, g$ на $\mathbb{N}$ такие, что $\mathrm{P}\{\xi=k+l\}=f(k) g(l)$. По лемме 4 (см. ниже) это функциональное уравнение допускает единственное решение вида $\mathrm{P}\{\xi=k\}=c \cdot q^{k}$, т.е. $\xi$ должна иметь геометрическое распределение.

ЛЕмма 4. Пусть для любых $k, l \in \mathbb{N}$ функиия $F: \mathbb{N} \rightarrow \mathbb{C}$ удовлетворяет соотношениям $F(k+l)=g(k) h(l)$ при подходящем выборе функиий $g, h$ на $\mathbb{N}$. Тогда существуют $c, q \in \mathbb{C}$ такие, что $F(n)=c \cdot q^{n}$.

ДокАЗАТЕЛЬСТво. Положим $m=\min \{k: F(k)=0\}$. Если $m<\infty$, то $F(m)=0$. Так как $m$ является минимумом, в этом случае не существует $k<m$ таких, что $g(k)=0$ или $h(k)=0$. Следовательно, не могут существовать $k, l<m$ такие, что $m=k+l$. Это влечет либо $m=0$, либо $m=1$. В обоих случаях утверждение леммы вьполняется. Таким образом, можно ограничиться случаем $F(k), g(k), h(k) \neq 0$ при всех $k \in \mathbb{N}$.

Для любых $k, l \in \mathbb{N}$ имеем

$$
1=\frac{F(k+l)}{F(k+l)}=\frac{g(k) h(l)}{h(k) g(l)}=\frac{g(k)}{h(k)} \frac{h(l)}{g(l)},
$$

а это показывает, что $g$ и $h$ могут отличаться лишь множителем. Поменяв их местами, можно сделать вьвод, что они должны равняться некоторой функции $f$. Тогда для любых $k \in \mathbb{N}$ имеем

откуда видно, что

$$
\frac{F(k+1)}{f(0)}=\frac{f(1)}{f(0)} f(k)=\frac{f(1)}{f(0)} \frac{F(k)}{f(0)},
$$

$$
F(k)=f(0)^{2}\left(\frac{f(1)}{f(0)}\right)^{k} .
$$

Лемма доказана.

Нормальное состояние $\widetilde{\omega}$ на $\mathfrak{L}(\Gamma \otimes \Gamma)$ назьвается мультипликативным, если

$$
\widetilde{\omega}(A \otimes B)=\widetilde{\omega}(A \otimes \mathbf{1}) \cdot \widetilde{\omega}(\mathbf{1} \otimes B)
$$

для произвольных $A, B \in \mathfrak{L}(\Gamma)$. Это свойство можно трактовать как независимость $\mathfrak{L}(\Gamma) \otimes \mathbf{1}$ и $\mathbf{1} \otimes \mathfrak{L}(\Gamma)$ относительно $\omega$. Несмотря на то, что гиббсовские состояния после расщепления имеют независимые энергетические уровни, сами расщепленные состояния не являются независимыми. Какие же состояния остаются независимыми после применения введенной вьше процедуры расщепления?

Поставим в соответствие каждому $r \in \mathbb{C},|r|<1$, вектор $\varphi_{r} \in \Gamma$ по правилу $\varphi_{r}(n)=r^{n}$. Определим соответствующее чистое фазовое состояние $\varphi^{r}$ как

$$
\varphi^{r}(\cdot)=\left(1-|r|^{2}\right)\left\langle\varphi_{r}, \cdot \varphi_{r}\right\rangle .
$$


ТЕОРемА 2. Пусть $\omega$ является нормальным состоянием на $\mathfrak{L}(\Gamma)$ таким, что для любых $A, B \in \mathfrak{L}(\Gamma)$ и некоторого $q, 0<q<1$, справедливо

$$
\omega\left(\mathscr{V}_{q}^{*}(A \otimes B) \mathscr{V}_{q}\right)=\omega\left(\mathscr{V}_{q}^{*}(A \otimes \mathbf{1}) \mathscr{V}_{q}\right) \cdot \omega\left(\mathscr{V}_{q}^{*}(\mathbf{1} \otimes B) \mathscr{V}_{q}\right)
$$

Тогда существует комплексное число $r \in \mathbb{C},|r|<1$, для которого $\omega=\varphi^{r}$. обратно, все фазовые состояния удовлетворяют соотношению (1).

ЗАмЕчаниЕ 6. В работе [15, теорема 15] (см. также [16], [17]) доказан этот же результат для случая независимого расщепления, когда состояние оказьвается когерентным. Существует аналогичный результат [3] для точечных процессов в случае независимого расшепления. В настоящее время неизвестны примеры точечных процессов, противоречашие теореме 2 .

ТЕОРемА 3. Имеется следующая связь между фазовыми и осиилляторными гиббсовскими состояниями:

$$
\omega^{\beta}=\frac{1}{2 \pi} \int_{0}^{2 \pi} d t \varphi^{e^{-\beta-i t}}
$$

Ввиду того, что смешивание по фазам не влияет на распределение расположений, независимость энергетических уровней расщепленного состояния $\omega^{\beta}$ становится очевидным следствием теоремы 2.

ДокАЗАТЕЛЬСТво. Для того чтобы доказать достаточность, рассмотрим ядро $\varrho$ состояния $\omega$. Тогда для ядра $\varrho^{\prime}$ расщепленного состояния $\omega\left(\mathscr{V}_{q}^{*}(\cdot) \mathscr{V}_{q}\right)$ справедливо представление

$$
\varrho^{\prime}\left(n_{1}, m_{1}, n_{2}, m_{2}\right)=\alpha_{q}\left(n_{1}, m_{1}\right) \alpha_{q}\left(n_{2}, m_{2}\right) \varrho\left(n_{1}+m_{1}, n_{2}+m_{2}\right) .
$$

Уравнение независимости (1) дает тождество

$$
\varrho\left(n_{1}+m_{1}, n_{2}+m_{2}\right)=f\left(n_{1}, n_{2}\right) g\left(m_{1}, m_{2}\right)
$$

при подходящем выборе $f$ и $g$. Применяя лемму 4 дважды, получаем требуемое утверждение. Необходимость доказьвается элементарно путем вьписьвания ядра расщепленного фазового состояния.

\section{СПИСОК ЦИТИРОВАННОЙ ЛИТЕРАТУРЫ}

[1] Landau L. D., Lifschitz E. M. Lehrbuch der theoretischen Physik. Bd. V. Statistische Physik Teil1. 2nd edition. Berlin: Akademie-Verlag, 1984.

[2] Lenk R. Einführung in die statistische Physik. Berlin: VEB Deutscher Verlag der Wissenschaften, 1978.

[3] Fichtner K.-H. Charakterisierung Poissonscher zufälliger Punktfolgen und infinitesimale Verdünnungsschemata // Math. Nachr. 1975. V. 68. P. 93-104.

[4] Campos R. A., Saleh B.E. A., Teich M. C. Quantum-mechanical lossless beam splitter: SU(2) symmetry and photon statistics // Phys. Rev. A (3). 1989. V. 40. №3. P. 1371-1384.

[5] Jeffers J. R., Imoto N., Loudon R. Quantum optics of travelling-wave attenuators and amplifiers // Phys. Rev. A (3). 1993. V. 47. №4. P. 3346-3359. 
[6] Fichtner K.-H., Freudenberg W., Liebscher V. Beam Splittings and Time Evolutions of Boson Systems. Preprint Math/Inf/96/39. Jena: Forschungsergebnisse der Fakultät für Mathematik und Informatik, 1996.

[7] Freudenberg W. On a class of quantum Markov chains on the Fock space // Quantum Probability \& Related Topics / ed. L. Accardi. V. XI. Singapore: World Sci. Publishing, 1994. P. 215-237.

[8] Fichtner K.-H., Freudenberg W. Point processes and the position distribution of infinite boson systems // J. Statist. Phys. 1987. V. 47. P. 959-978.

[9] Vourdas A. Phase States: An analytical approach in the unit disc // Phys. Scripta. 1993. V. 48. P. $84-86$.

[10] Perelomov A. M. Generalized Coherent States and Their Applications. Texts Monographs Phys. Berlin-Heidelberg-New York: Springer, 1986.

[11] Emch G. G. Mathematical and Conceptual Foundations of 20th Century Physics. NorthHolland Math. Stud. V. 100. Amsterdam-New York-Oxford: North-Holland, 1984.

[12] Parthasarathy K. R. An Introduction to Quantum Stochastic Calculus. Basel-BostonBerlin: Birkhäuser, 1992.

[13] Fichtner K.-H., Freudenberg W. Characterization of states of infinite boson systems. I. On the construction of states of boson systems // Comm. Math. Phys. 1991. V. 137. P. 315-357.

[14] Ferguson T.S. A characterization of the geometric distribution // Amer. Math. Monthly. 1965. V. 72. P. 256-260.

[15] Fichtner K.-H., Freudenberg W., Liebscher V. Characterization of coherent and mixed coherent states by beam splittings (to appear).

[16] Liebscher V. Characterization of Coherent and Mixed Coherent States by Beam Splittings. Preprint Math/Inf/96/16. Jena: Forschungsergebnisse der Fakultät für Mathematik und Informatik, 1996.

[17] Titulaer U. M., Glauber R. J. Density operators for coherent fields // Phys. Rev. 1966. V. 145. № 4. P. 1041-1050.

Friedrich-Schiller-Universität, Jena (Germany)

Поступило

Brandenburgische Technische Universität Cottbus, Cottbus (Germany) 16.09.97 